J Phys Chem B. 2006 February 9; 110(5): 2150-2154. doi:10.1021/jp056606x.

\title{
Multipole Plasmon Resonances in Gold Nanorods
}

\author{
Emma Kathryn Payne, \\ Department of Chemistry and International Institute for Nanotechnology, Northwestern University, \\ 2145 Sheridan Road, Evanston, IL 60208-3113 (USA), Fax: (+1) 847-467-5123
}

\section{Kevin L. Shuford,}

Department of Chemistry and International Institute for Nanotechnology, Northwestern University, 2145 Sheridan Road, Evanston, IL 60208-3113 (USA)

\section{Sungho Park, \\ Department of Chemistry \& Sungkyunkwan Advanced Institute of Nanotechnology, Sungkyunkwan University, Suwon 440-746 (Korea)}

Prof. George C. Schatz, and

Department of Chemistry and International Institute for Nanotechnology, Northwestern University, 2145 Sheridan Road, Evanston, IL 60208-3113 (USA)

\section{Prof. Chad A. Mirkin}

Department of Chemistry and International Institute for Nanotechnology, Northwestern University, 2145 Sheridan Road, Evanston, IL 60208-3113 (USA), Fax: (+1) 847-467-5123

Chad A. Mirkin: chadnano@northwestern.edu

\section{Abstract}

The optical properties of gold rods electrochemically deposited in anodic aluminum oxide templates have been investigated. Homogeneous suspensions of rods with average diameter of 85 $\mathrm{nm}$ and varying lengths of $96,186,321,465,495,578,641,735$, and $1175 \mathrm{~nm}$ were fabricated. The purity and dimensions of these rod nanostructures allowed us to observe higher order multipole resonances for the first time in a colloidal suspension. The experimental optical spectra agree with discrete dipole approximation calculations that have been modeled from the dimensions of the gold nanorods.

\section{Keywords}

gold nanorods; multipoles; anodic aluminum oxide template; discrete dipole approximation

\section{Introduction}

Metal nanostructures have been of significant interest as a result of their many uses in photonics ${ }^{1}$, electronics ${ }^{2}$, biodetection ${ }^{3}$, and catalysis. ${ }^{4}$ These materials have unique optical and chemical properties that are quite different from the bulk or atomic species. Gold and silver nanoparticles are particularly interesting because their plasmon resonance excitation gives rise to intense colors, Raman enhancing capabilities, and their utility as labels in biodetection platforms. The wavelength of the plasmon resonance can be tuned by the size

Correspondence to: Chad A. Mirkin, chadnano@northwestern.edu.

Supporting Information Available: SEM image of anodic aluminum template. Extinction spectra and SEM images of other size gold rods. This material is available free of charge via the Internet at http://pubs.acs.org. 
and shape of the nanoparticle, and by its surrounding dielectric medium. ${ }^{5}$ For example, when a spherical nanoparticle's diameter is approximately $5-50 \mathrm{~nm}$, the plasmon resonance is dipolar in nature because the conduction electrons in the metal are excited in-phase with the incident electromagnetic field. However, when the dimensions are larger, the excitation of higher order harmonics or multipoles can be excited ${ }^{5}$ as a result of phase retardation of the applied field inside the material. Such multipole resonances are of fundamental interest, and the near-field profiles and the far-field scattering patterns of multipolar harmonics can be useful in applications pertaining to light signal routers, light manipulators, or multi-step enhancers in processes like second harmonic generation. ${ }^{6}$ In addition, higher order modes provide a spectroscopic fingerprint of the material that can be used to characterize and assess the quality of such structures.

In past work quadrupole and higher order multipoles have been detected in both isotropic and anisotropic gold or silver materials including shells, ${ }^{6}$ spheres, ${ }^{7}$ and prisms. ${ }^{8}$ The multipoles also have been observed in lithographically generated analogs of gold and silver platelets, ${ }^{9}$ but no one has generated free-standing rods in the form of a colloid with spectroscopically observed higher order resonances. Herein we present the first experimental observation of higher order surface plasmon modes for colloidal cylindrical gold rods. In addition we use discrete dipole approximation (DDA) calculations to identify the number of expected modes for the size rods studied.

Typically gold nanorods are synthesized via wet-chemical techniques ${ }^{10}$ or hard templatedirected methods, ${ }^{11}$ and have received much attention for their potential use in therapeutics, ${ }^{12,13}$ chemical separations, ${ }^{14}$ sensing, ${ }^{12,15}$ and optics. ${ }^{12,10}$ In the wet-chemical synthesis, metal ions are reduced onto the surface of gold seeds in the presence of various surfactants. ${ }^{13}$ The resulting shape is dependent on the reaction conditions, seed, and surfactant or capping agent used. Although this technique proved to be useful in terms of controlling the length of nanorods, usually the rod diameter varied from $\sim 5$ to 55 $\mathrm{nm} .{ }^{15 \mathrm{a}, 16,17}$ Therefore, only two surface plasmon resonance peaks have been observed. One is parallel to the short axis of the rod (transverse mode) and is typically in the range of 500 to $550 \mathrm{~nm}$, while the other is parallel to the long axis (longitudinal mode) and is substantially more variable with the plasmon wavelength depending on the length of the rods. In addition, this wet-chemical method also can produce spherical particles or other shape materials as byproducts, which can greatly affect the optical response. Therefore, if one is interested in mapping out the fundamental optical properties of high purity rod structures, hard template-directed synthesis that utilize polycarbonate membranes and anodic aluminum oxide (AAO) templates, ${ }^{11}$ at present provide a more reliable means of preparing such structures than the solution phase methods. Herein we show that these AAO templates provide access to rod structures that exhibit higher order surface plasmon resonances, which have never been observed in the context of a colloid.

\section{Experimental Section}

\section{AAO Fabrication}

Following a two-step anodization process similar to that established by Masuda and coworkers, ${ }^{18}$ we synthesized an AAO template with $85 \pm 10 \mathrm{~nm}$ diameter pores (Supporting Information Figure 1). During this experimental set-up, aluminum served as the anode while graphite served as the cathode in the electrochemical cell. A high-purity $(99.999 \%)$ thin sheet of aluminum (PVD Materials) was first electropolished in a mixture of ethanol (Aldrich) and perchloric acid (Aldrich) $(8: 1, \mathrm{v} / \mathrm{v})$ at $15 \mathrm{~V}$ in order to reduce the surface roughness of the aluminum. Then the smooth aluminum was anodized in $0.3 \mathrm{M}$ oxalic acid (Acros) at $40 \mathrm{~V}$ and $1^{\circ} \mathrm{C}$ for $20 \mathrm{~h}$ to initiate the growth of the porous alumina. The alumina was next removed using an aqueous mixture of chromic acid (Aldrich) $(1.8 \mathrm{wt} \%)$ and 
phosphoric acid (Aldrich) (6 wt \%). A second anodization followed in $0.3 \mathrm{M}$ oxalic acid at $40 \mathrm{~V}$ and $1^{\circ} \mathrm{C}$ for $24 \mathrm{~h}$ to insure highly ordered porous alumina. The remaining aluminum was subsequently removed in a saturated $\mathrm{HgCl}_{2}$ (Aldrich) solution resulting in porous $\mathrm{AAO}$ templates. These templates were then subjected to a $6 \mathrm{wt} \%$ phosphoric acid etchant for pore widening.

\section{Gold Rod Synthesis}

Gold nanorods were electrochemically deposited in the pores of the AAO template following a similar protocol pioneered by Martin and Moskovits. ${ }^{11}$ A layer of silver $(200 \mathrm{~nm})$ was evaporated on one side of the AAO. After making a contact with aluminum foil in a Teflon cell, this material served as a cathode in the electrochemical cell, in which $\mathrm{Ag} / \mathrm{AgCl}$ served as a reference electrode and platinum wire acted as a counter electrode. The nanopores were filled with $\mathrm{Ag}$ plating solution (Technic Inc.) at a constant potential, $-0.9 \mathrm{~V}$ vs $\mathrm{Ag} / \mathrm{AgCl}$ followed by $\mathrm{Au}$ plating solution (Technic Inc.) also at $-0.9 \mathrm{~V}$ vs $\mathrm{Ag} / \mathrm{AgCl}$.

The lengths of the rods were controlled by monitoring the number of coulombs passed during the deposition process. The rods were then released from the template in a $3 \mathrm{M} \mathrm{NaOH}$ solution, rinsed four times with water, two times with ethanol, and then resuspended in $\mathrm{D}_{2} \mathrm{O}$ so that the near infrared (NIR) optical properties of the rods could be observed. The rods were suspended via sonication and no surfactant or stabililizer was used.

\section{Materials Characterization}

Scanning electron microscopy (SEM) images were taken on a Leo 1525 courtesy of Northwestern University Nuance Facility. Experimental extinction spectra did not use polarized light and were collected on a Cary 5000 UV-vis-NIR spectrophotometer.

\section{Results}

We were able to synthesize rods with an average diameter of $85 \mathrm{~nm}$ and average lengths of 96 (Figure 1A), 186, 321, 465, 495, 578, 641 (Figure 1B), 735 (Figure 1C), and $1175 \mathrm{~nm}$ (Figure 1D). Scanning electron microscopy (SEM) images and histograms reveal that only rod shaped materials with a narrow size distribution are present in the solution, Figure 1.

The optical properties of these rods were investigated using UV-vis-NIR spectroscopy. When the length is $96 \mathrm{~nm}$, one prominent broad peak around $600 \mathrm{~nm}$ is observed, which is indicative of both the transverse and longitudinal dipole modes, Figure 2A. The longitudinal plasmon, labeled I, overlaps the transverse dipole mode because the aspect ratio (length over diameter) is close to 1 , and the rod spectrum resembles that of a near isotropic sphere or disc. However, Gans theory predicts that when the aspect ratio increases, the longitudinal mode will red-shift to longer wavelengths and the transverse mode will blue-shift to slightly shorter wavelengths. ${ }^{19}$ This trend was observed in gold nanorods made via solution phase synthesis. ${ }^{15,17}$ The gold nanorods synthesized via the hard template-directed method exhibit a similar trend, Figure 2 and (Supporting Information Figure 2). One particularly interesting feature is the appearance of both even (labeled II, IV, VI) and odd (labeled I, III, V, VII) higher order multipole resonances when the aspect ratio is greater than 4, Figure 2B-D. These multipole resonances have not been observed previously in a colloidal suspension of gold nanorods and are assigned based on theoretical calculations which are discussed in further detail below.

When increasing the rod aspect ratio, both the even and odd higher order longitudinal modes (II-VII) red-shift to longer wavelengths, Figure 2B-D. Another noticeable feature is the increase in the number of multipole resonances. When the length of the rod reaches $641 \mathrm{~nm}$, the transverse dipole mode, labeled * in Figure 2B appears at $530 \mathrm{~nm}$ while two other higher 
order modes, II and III, are observed at $846 \mathrm{~nm}$ and $1197 \mathrm{~nm}$ respectively. In this size regime the longitudinal dipole mode, I, is red-shifted to longer wavelengths and not detectable in the spectral range studied. Also a small shoulder is visible around $660 \mathrm{~nm}$, which corresponds to the onset of multipole IV. Again, as the aspect ratio increases, the transverse mode remains around $530 \mathrm{~nm}$, while the higher order modes red-shift and increase in intensity and number, Figure $2 \mathrm{C}$. When the length is $735 \mathrm{~nm}$, the II, III, and IV multipoles are at $1397 \mathrm{~nm}, 966 \mathrm{~nm}, 775 \mathrm{~nm}$, respectively, and multipole $\mathrm{V}$ appears at around $660 \mathrm{~nm}$. This same trend is followed in the spectrum for the rods that are $1175 \mathrm{~nm}$ long and up to seven multipoles are detected, Figure 2D. In the spectra each higher order mode is resolved from the transverse mode at around 650 to $700 \mathrm{~nm}$. The minimum at around 800 $\mathrm{nm}$ is due to detector and lamp changeover in the instrument. The assignments in Figure 2 are based upon the characterization of these rods by SEM and electrodynamics results which we discuss below.

\section{Electrodynamics Calculations}

We have studied the optical properties of the gold rods using the discrete dipole approximation (DDA) method. In this method, ${ }^{20}$ the particle is represented as a cubic array of point dipoles. The polarization of each dipole arises in response to the total electromagnetic field at that site in the array as described by

$$
\stackrel{\rho}{P}=\alpha_{j}\left[\begin{array}{l}
\rho \text { inc } \\
E_{j}
\end{array}-\sum_{k \neq j}^{N} A_{j k} \stackrel{\rho}{P_{k}}\right]
$$

where $\alpha$ is the polarizability, ${ }_{E}^{i \text { inc }}$ is the incident field, and $A$ is an interaction matrix. The polarizability is determined by the dielectric constant of gold (here we have used values from Johnston and Christy ${ }^{21}$ ) using a lattice dispersion relation. ${ }^{22}$ The total field is the superposition of an incident plane wave, and the fields radiating from all of the other dipoles in the array given by

$$
A_{j k} \stackrel{\rho}{P_{k}}=\frac{\exp \left(i k r_{j k}\right)}{r_{j k}^{3}}\left\{k^{2} \stackrel{\rho}{r}_{j k} \times\left(\stackrel{\rho}{r}_{j k} \times \stackrel{\rho}{P} k\right)+\frac{\left(1-i k r_{j k}\right)}{r_{j k}^{2}}\left[r_{j k}^{2} \stackrel{\rho}{P}{ }_{k}-3 r_{j k}^{\rho}\left({ }_{j k}^{\rho} \cdot \stackrel{\rho}{P_{k}}\right)\right]\right\} .
$$

Assigning the diagonal elements of matrix $A$ to be $\alpha_{j}^{-1}$ permits rearrangement of Eq. (1) to

$$
\sum_{k=1}^{N} A_{j k} \stackrel{\rho}{P_{k}}=\stackrel{\rho}{E_{j}}
$$

where $P$ and $E_{\text {inc }}$ are $3 \mathrm{~N}$-dimensional vectors and $A$ is a $3 \mathrm{~N} \times 3 \mathrm{~N}$ matrix. For the number of dipoles necessary to represent large nanoparticles, direct matrix inversion to obtain $P$ is not feasible, so an iterative solution is utilized to find the polarization of each dipole. Once the dipole polarizations are known, the extinction cross section can be computed using 


$$
C_{\text {ext }}=\frac{4 \pi k}{\left.\left|\frac{\rho}{E}\right|^{i n c}\right|^{2}} \sum_{j=1}^{N} \operatorname{Im}\left\{\begin{array}{ll}
\rho^{i n c, *} & \rho \\
E_{j} & \cdot
\end{array}\right\} .
$$

An important consideration in making comparison of theory and experiment concerns the effect of averaging over orientation of the rod relative to the polarization vector. Figure 3 shows DDA results for a rod that is $1175 \mathrm{~nm}$ in length and $85 \mathrm{~nm}$ in diameter as a function of the angle $\theta$ between the rod axis and the polarization vector. $\theta=90$ refers to transverse excitation (Figure 3A) and we see the expected resonance at $580 \mathrm{~nm} . \theta=0$ is for longitudinal excitation (Figure 3E) and this shows that only odd order modes (labeled III, V, VII as mode I is at longer than $2 \mu \mathrm{m}$ ) are excited in this case. The suppression of the even modes results from symmetry of the induced polarization when the wavevector is perpendicular to and the polarization is parallel to the rod axis. This leads to an induced polarization that is antisymmetric with respect to reflection through a plane that bisects the rod, and only odd-order modes are excited. The intermediate angles (Figure 3B-D) show that a mixture of transverse and longitudinal excitations is required to see all the multipolar resonances. In particular the resonances are at 1400, 1090, 900, 780 and $690 \mathrm{~nm}$ for modes III, IV, V, VI and VII, respectively. Note that in past work with lithographically fabricated rectangular rods on surfaces, the polarization was only chosen to be in the longitudinal direction so only the odd-order modes were observed. ${ }^{9 b}$

In addition, the plot of $\lambda$ max vs. aspect ratio in Figure 4 further supports the multipole assignments in Figure 2. The graph illustrates where multipoles I, II, III, IV (Figure 4A-D) are expected for varying lengths of gold rods with $85 \mathrm{~nm}$ diameters. The All theoretical plots show a good linear fit with $\mathrm{R}^{2}$ values of 0.99 and provide linear equations for determining where the multipoles appear in the infrared for $85 \mathrm{~nm}$ diameter gold rods in solution. In addition the experimental plots (filled shapes) agree well with the calculated plots.

Therefore, the multipole assignments in the experimental spectra are correctly labeled.

\section{Comparison of Theory and Experiment}

Figure 5 presents a comparison of experimentally measured spectra and DDA results. The extinction spectra are in generally good agreement. For example, the theoretical and experimental extinction spectra for $96 \mathrm{~nm}$ long gold rods have one broad peak at $\sim 600 \mathrm{~nm}$, Figure 5A. The spectra for the $641 \mathrm{~nm}$ long rods, Figure 5B, both exhibit three higher order plasmon resonances at approximately $685 \mathrm{~nm}, 895 \mathrm{~nm}$, and $1200 \mathrm{~nm}$ and a transverse mode at $\sim 530 \mathrm{~nm}$. Comparing the spectra for the $735 \mathrm{~nm}$ long rods, Figure 5C, a transverse mode is observed at $530 \mathrm{~nm}$ and $571 \mathrm{~nm}$ for the experimental and theoretical spectra, respectively. Four more multipole resonances are also evident in both spectra at 650, 760, 960, and 1400 $\mathrm{nm}$ wavelengths. In addition both the theoretical and experimental spectra for the $1175 \mathrm{~nm}$ long rods, Figure 5D, show a transverse band around $550 \mathrm{~nm}$ and higher order plasmon resonances VII, VI, V, IV, and III at 680, 760, 875, 1060, and $1410 \mathrm{~nm}$, respectively. The differences between the transverse and longer wavelength multipoles are due to the size distribution in both the length and diameter of the nanorods in the experimental samples, Figure 1. However, the theoretical spectra for all the different aspect ratio rods agree well with the experimental data both quantitatively and qualitatively in that both the theoretical and experimental spectra for all aspect ratios have the same number of multipoles at similar wavelengths. 


\section{Conclusion}

In conclusion we have observed multiple higher order plasmon resonances in colloidal cylindrical gold nanorods for the first time. The AAO template provided a synthetic route that resulted in a homogeneous suspension of rods with the proper dimensions to observe these modes. As in the lithographically generated patterns, ${ }^{10}$ both the even and odd modes were detected up to the $7^{\text {th }}$ order and were in good agreement with DDA calculations. Unlike the lithographically generated patterns, ${ }^{10}$ these rods are not dependent on surface selection rules, and both even and odd multipoles can be observed. These results are of significant fundamental importance and could potentially impact the aforementioned applications. Perhaps the most important contribution is a set of benchmark spectra for evaluating the quality of a colloid of gold rods prepared in any manner. Solution spectra can be quite informative, and the presence of the multipole resonances is a signature of a high quality preparatory procedure that will guide other researchers in determining the homogeneity of their anisotropic structures. In addition, this synthetic technique enables the fabrication of multi-component nanorods that can provide multiple surface plasmon resonances for applications in which solution phase methods and lithographically generated patterns are limited.

\section{Supplementary Material}

Refer to Web version on PubMed Central for supplementary material.

\section{Acknowledgments}

This work was supported by the NSF-NSEC, the ONR, and the AFOSR. GCS is grateful for support from the DOE (DEFGO2-02-ERIS 487). CAM acknowledges a NIH Director's Pioneer Award.

\section{References}

1. Synthesis and Plasmonic Properties of Nanostructures. Mater Res Bull. 2005:30.

2. (a) Duan X, Huang Y, Cui Y, Wang J, Lieber CB. Nature. 2001; 409:66. [PubMed: 11343112] (b) Huang Y, Duan X, Wei Q, Lieber CB. Science. 2001; 291:630. [PubMed: 11158671] (c) Cobden DH. Nature. 2001; 409:32. [PubMed: 11343099] (d) Tseng GY, Ellenbogen JC. Science. 2001; 294:1293. [PubMed: 11701916] (e) Service RF. Science. 2001; 293:782. [PubMed: 11486066] (f) Park S, Chung S-W, Mirkin CA. J Am Chem Soc. 2004; 126:11772. [PubMed: 15382894] (g) Kovtyukhova NI, Kelley BK, Mallouk TE. J Am Chem Soc. 2004; 126:12738. [PubMed: 15469244] (h) Kovtyukhova NI, Mallouk TE. Adv Mater. 2005; 17:187.(i) Quin L, Park S, Huang L, Mirkin CA. Science. 2005; 309:113. [PubMed: 15994551]

3. (a) Mirkin CA, Letsinger RL, Mucic RC, Storhoff JJ. Nature. 1996; 382:607. [PubMed: 8757129] (b) Alivisatos AP, Johnsson KP, Peng X, Wilson TE, Loweth CJ, Bruchez MP Jr, Schultz PG. Nature. 1996; 382:609. [PubMed: 8757130] (c) Taton TA, Mirkin CA, Letsinger RL. Science. 2000; 289:1757. [PubMed: 10976070] (d) Park SJ, Taton TA, Mirkin CA. Science. 2002; 295:1503. [PubMed: 11859188] (e) Cao YC, Jin R, Mirkin CA. Science. 2002; 297:1536. [PubMed: 12202825] (f) Nam JM, Thaxton CS, Mirkin CA. Science. 2003; 301:1884. [PubMed: 14512622] (g) Katz E, Willner I. Angew Chem. 2004; 116:6166.Angew Chem Int Ed. 2004; 43:6042.(h) Nam JM, Stoeva SI, Mirkin CA. J Am Chem Soc. 2004; 126:5932. [PubMed: 15137735] (i) Georganopoulou DG, Chang L, Nam JM, Thaxton CS, Mufson EJ, Klein WL, Mirkin CA. Proc Natl Acad Sci U S A. 2005; 102:2273. [PubMed: 15695586] (j) Rosi NL, Mirkin CA. Chem Rev. 2005; 105:1547. [PubMed: 15826019]

4. (a) Martin CR. Adv Mater. 1991; 3:457.(b) Bonnemann H, Richards RM. Eur J Inorg Chem. 2001:2455.(c) Bell AT. Science. 2003; 299:1688. [PubMed: 12637733] (d) Zhang H, Guo Y, Wan L, Bai CL. Chem Commun. 2003; 24:3022.(e) Burda C, Chen X, Narayanan R, El-Sayed MA. Chem Rev. 2005; 105:1025. [PubMed: 15826010] 
5. (a) Bohren, CF.; Huffman, DR. Absorption and Scattering of Light by Small Particles. John Wiley \& Sons; New York: 1998. (b) Mulvaney P. Langmuir. 1996; 12:788.(c) Kreibig U, Schmitz B, Brewer HD. Phys Rev B. 1987; 36:5027.(d) Ruppin, R. Electromagnetic Surface Modes. John Wiley \& Sons; New York: 1982. (e) Ruppin R. Phys Rev B. 1975; 11:2871.(f) Clagnet R. Optik. 1972; 35:180.(g) Doremus R. J Chem Phys. 1965; 42:414.

6. Oldenburg SJ, Jackson JB, Westcott SL, Halas N. J Appl Phys Lett. 1999; 75:2897.

7. (a) Kumbhar AS, Kinnan MK, Chumanov G. J Am Chem Soc ASAP. 2005(b) Evanoff DD Jr, Chumanov G. J Phys Chem B. 2004; 108:13948.(c) Evanoff DD Jr, Chumanov G. J Phys Chem B. 2004; 108:13957.(d) Malynych S, Chumanov G. J Amer Chem Soc. 2003; 125:2896. [PubMed: 12617655]

8. (a) Hulteen JC, Van Duyne RP. J Vac Sci Technol A. 1995; 13:1553.(b) Jin R, Cao Y, Mirkin CA, Kelly KL, Schatz GC, Zheng JG. Science. 2001; 294:1901. [PubMed: 11729310] (c) Jin R, Cao YC, Mirkin CA. Nature. 2003; 425:487. [PubMed: 14523440] (d) Millstone JE, Park S, Shuford KL, Qin L, Schatz GC, Mirkin CA. J Am Chem Soc. 2005; 127:5312. [PubMed: 15826156]

9. (a) Krenn JR, Schider G, Rechberger W, Lamprecht B, Leitner A, Aussenegg FR, Weeber JC. Appl Phys Letts. 2000; 77:3379.(b) Schaich WL, Schider G, Krenn JR, Leitner A, Aussenegg FR, Puscasu I, Monacelli B, Boreman G. Applied Optics. 2003; 42:5714. [PubMed: 14528934] (c) Laurent G, Felidj N, Aubard J, Levi G, Krenn JR, Hohenau A, Schider G, Leitner A, Aussenegg FR. J Chem Phys. 2005; 122 on line. (d) Laurent G, Felidj N, Aubard J, Levi G, Krenn JR, Hohenau A, Schider G, Leitner A, Aussenegg FR. Physical Review B. 2005; 71(e) Hohenau A, Krenn JR, Schider G, Dilbacher H, Leitner A, Aussenegg FR, Schaich WL. Europhys Lett. 2005; 69:538.

10. (a) Li T, Hunyadi SE, Gou L, Gao J, Orendorff CJ, Gole AM, Sau T, Murphy CJ. J Phys Chem B. 2005; 109:13857. [PubMed: 16852739] (b) Soennichsen C, Alivisatos AP. Nano Letts. 2005; 5:301. [PubMed: 15794615]

11. (a) Penner RM, Martin CR. Anal Chem. 1987; 59:2625.(b) Martin CR. Adv Mater. 1991; 3:457.(c) Martin CR. Science. 1994; 266:1961. [PubMed: 17836514] (d) Martin CR. Chem Mater. 1996; 8:1739.(e) Routkevitch, Bigioni T, Moskovits M, Xu JM. J Phys Chem. 1996; 100:14037.(f) Thompson GE, Furneaux RC, Wood GC, Richardson JA, Gode JS. Nature. 1978; 272:433.(g) Diggle JW, Downie TC, Goulding CW. Chem Rev. 1968; 69:365.(h) Keller F, Hunter MS, Robinson DL. J Electrochem Soc. 1953; 100:411.

12. Perez-Juste J, Pastoriza-Santos I, Liz-Marzan LM, Mulvaney P. Coord Chem Revs. 2005; 249:1870.

13. (a) Salem AK, Searson PC, Leong KW. Nature Mater. 2003; 2:668. [PubMed: 12970757] (b) Salem AK, Hung CF, Kim TW, Wu TC, Searson PC, Leong KW. Nanotechnol. 2005; 16:484.

14. (a) Lee KB, Park S, Mirkin CA. Angew Chem. 2004; 116:3110.Angew Chem Int Ed. 2004; 43:3048.(b) Oh B-K, Park S, Lee SW, Lee K-B, Mirkin CA. unpublished results.

15. (a) Jana NR, Gearheart L, Murphy CJ. J Phys Chem B. 2001; 105:4065.(b) Kim F, Song JH, Yang P. J Am Chem Soc. 2002; 124:14316. [PubMed: 12452700] (c) Johnson CJ, Dujardin E, Davis SA, Murphy CJ, Mann S. J Mater Chem. 2002; 12:1765.(d) Nikoobakht B, El-Sayed MA. Chem Mater. 2003; 15:1957.

16. (a) Foss CA Jr, Hornyak GL, Stockert JA, Martin CR. J Phys Chem. 1994; 98:2963.(b) van der Zande BMI, Bohmer MR, Fokkink LGJ, Schonenberger C. J Phys Chem B. 1997; 101:852.(c) van der Zande BMI, Bohmer MR, Fokkink LGJ, Schonenberger C. Langmuir. 2000; 16:451.(d) van der Zande BMI, Dhont JKG, Bohmer MR, Philipse AP. Langmuir. 2000; 16:459.

17. (a) Link S, Burda C, Nikoohakht B, El-Sayed MA. J Phys Chem B. 2000; 104:6152.(b) Busbee BD, Obare SO, Murphy CJ. Adv Mater. 2003; 15:414.(c) Sau TK, Murphy CJ. Langmuir. 2004; 20:6414. [PubMed: 15248731] (d) Gou L, Murphy CJ. Chem Mater. 2005; 17:3668.

18. (a) Masuda H, Fukuda K. Science. 1995; 268:1466. [PubMed: 17843666] (b) Masuda H, Mizuno T, Baba N, Ohmori T. J Electroanal Chem. 1994; 368:333.(c) Masuda H, Nishio K, Baba N. Thin Solid Films. 1993; 223:1.

19. (a) Gans R. Ann Phys. 1912; 37:881.(b) Gans R. Ann Phys. 1915; 47:270.

20. (a) Draine BT, Flatau PJ. J Opt Soc Am A. 1994; 11:1491.(b) Purcell EM, Pennypacker CR. Astrophys J. 1973; 186:705.(c) Draine BT. Astrophys J. 1988; 333:848.

21. Johnson PB, Christy RW. Phys Rev B. 1972; 6:4370. 
22. Draine BT, Goodman J. Astrophys J. 1993; 405:685. 

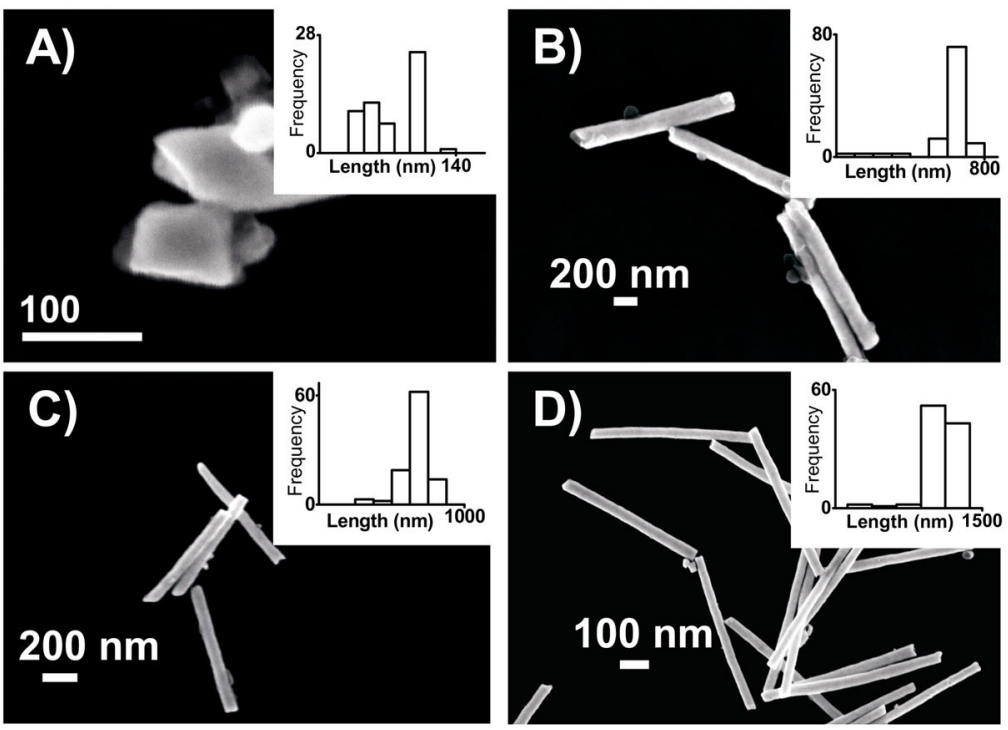

Figure 1.

SEM images of rods A) $96 \pm 18 \mathrm{~nm}$, B) $641 \pm 47 \mathrm{~nm}$, C) $735 \pm 48 \mathrm{~nm}$, and D) $1175 \pm 49 \mathrm{~nm}$ long with $85 \pm 10 \mathrm{~nm}$ diameters. All of these images and the corresponding histograms found in the insets illustrate the homogeneous nature of the gold rods in solution. 


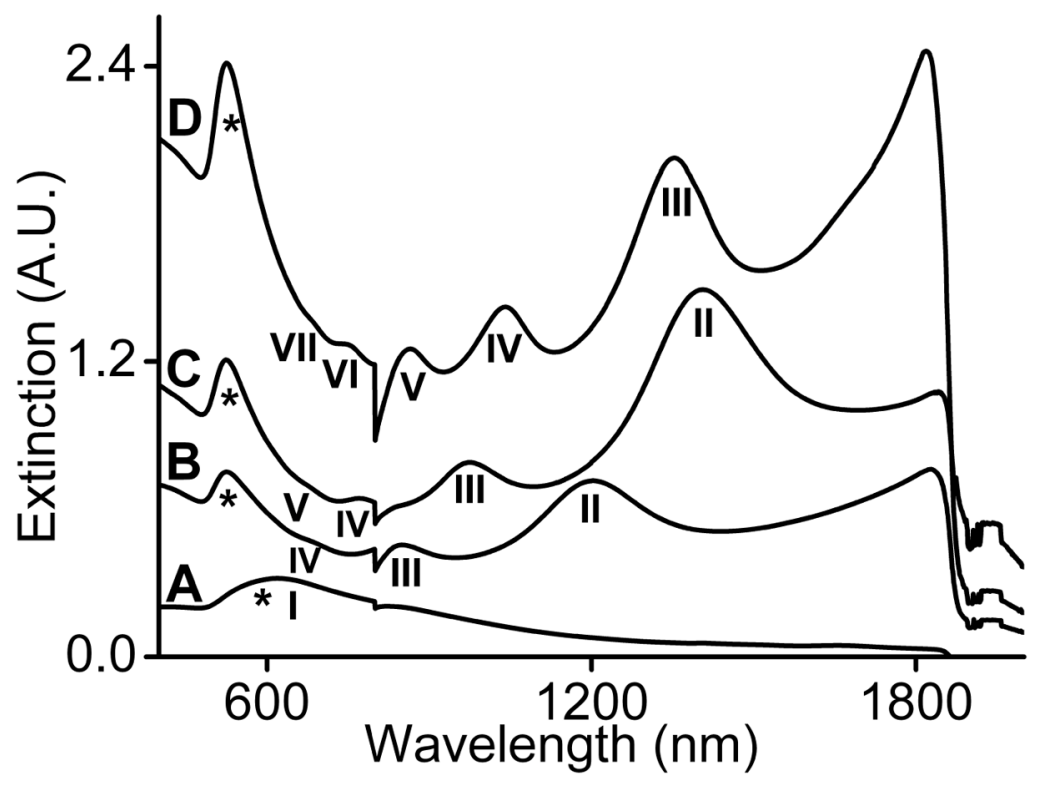

Figure 2.

UV-vis-NIR spectra of the A) 96, B) 641, C) 735 , and D) $1175 \mathrm{~nm}$ long gold rods in $\mathrm{D}_{2} \mathrm{O}$. The Roman numeral labels the multipole order associated with each plasmon resonance. Orders were assigned based on theoretical calculations. 


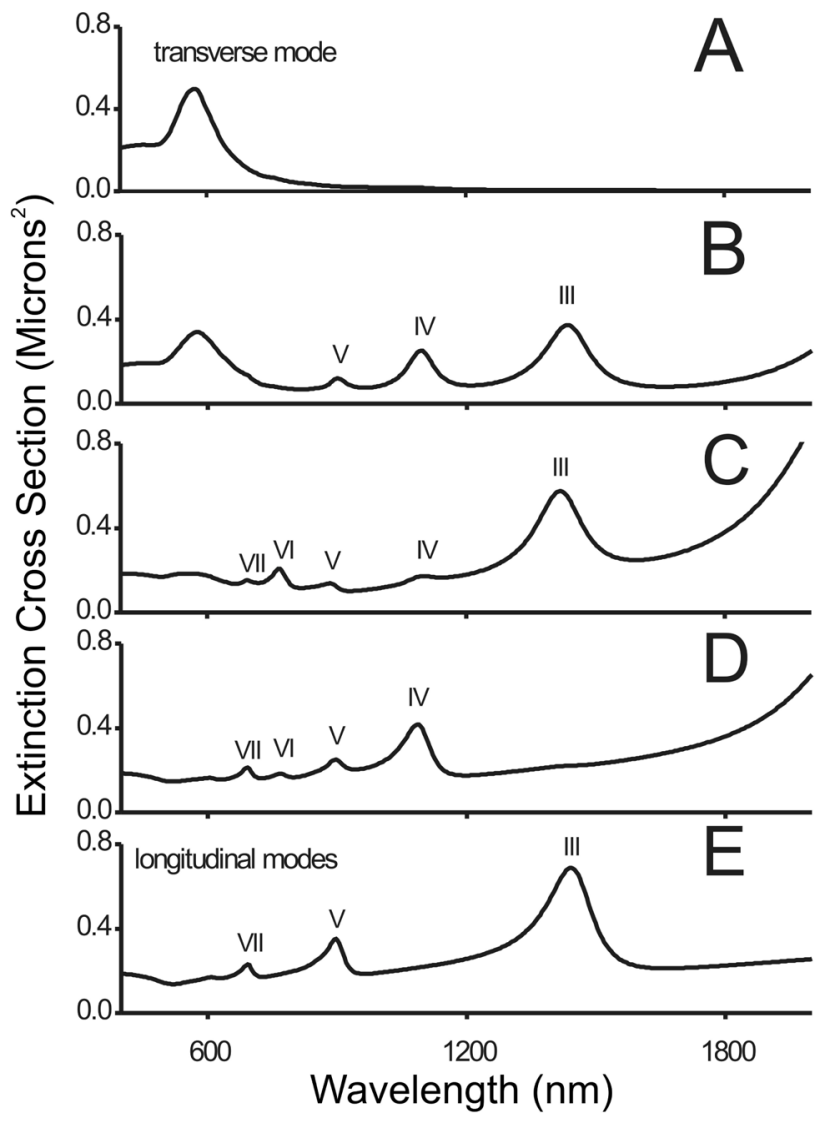

Figure 3.

Extinction spectra (from DDA calculations) for $1175 \mathrm{~nm}$ rods, showing the effect of varying the orientation of the rod relative the polarization direction. A) $\theta=90$, B) $\theta=67.5$, C) $\theta=$ 45 , D) $\theta=22.5$, E) $\theta=0$ where $\theta$ is the angle between the rod and polarization direction. 


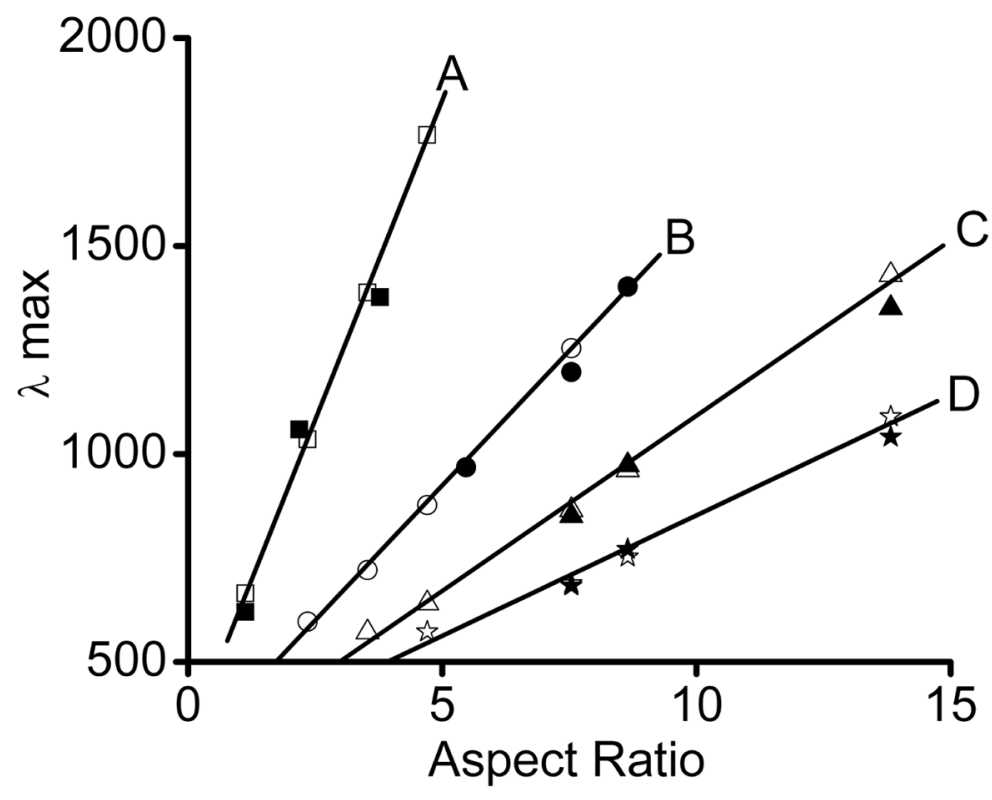

Figure 4.

Plot of $\lambda \max$ vs. aspect ratio for $85 \mathrm{~nm}$ diameter gold rods in solution. Theoretical points are represented by hollow shapes while experimental are represented by filled shapes for multipole A) I, B) II, C) III, and D) IV. 

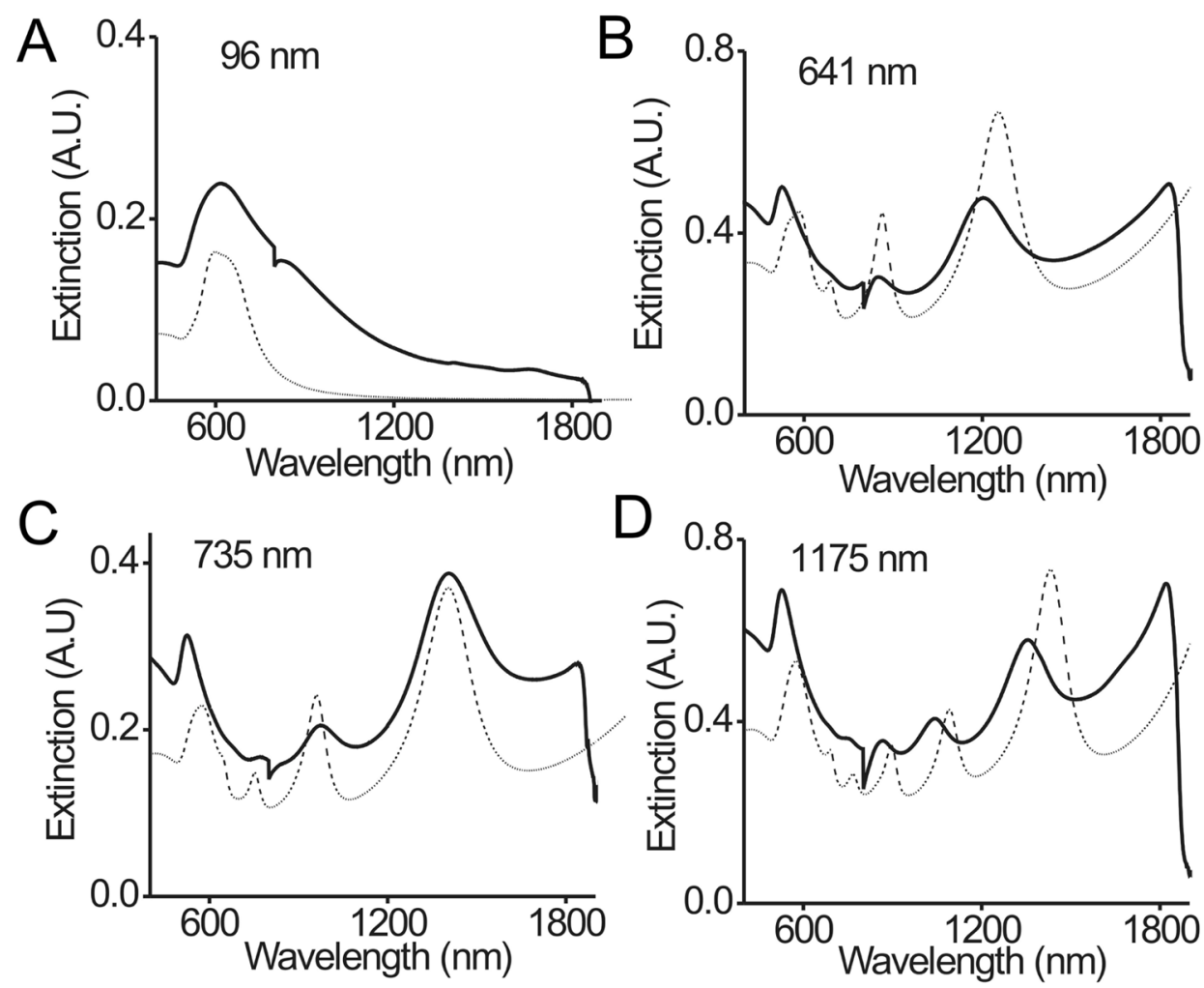

Figure 5.

Theoretical (DDA) extinction spectra (dashed line) compared to experimental extinction spectra (solid line) for gold rods with a fixed $85 \mathrm{~nm}$ diameter and A) 96, B) 641, C) 735, and D) $1175 \mathrm{~nm}$ lengths. 